\title{
AMENDMENTS
}

\section{Author Correction: Electric and antiferromagnetic chiral textures at multiferroic domain walls}

J.-Y. Chauleau, T. Chirac, S. Fusil, V. Garcia, W. Akhtar, J. Tranchida, P. Thibaudeau (D), I. Gross, C. Blouzon, A. Finco ID, M. Bibes (D), B. Dkhil (D), D. D. Khalyavin, P. Manuel, V. Jacques, N. Jaouen (D) and M. Viret (D)

Correction to: Nature Materials https://doi.org/10.1038/s41563-019-0516-Z, published online 4 November 2019.

In the version of this Letter originally published, in the Acknowledgements section the text 'V.J. acknowledges financial support by the European Research Council (ERC-StG-2014, Imagine)' should have read 'V.J. acknowledges financial support by the European Research Council (ERC-StG-2014, Imagine) and the EU Quantum Flagship project ASTERIQS (820394)'. This has now been corrected in all versions of the Letter.

Published online: 12 November 2019

https://doi.org/10.1038/s41563-019-0558-2

(c) The Author(s), under exclusive licence to Springer Nature Limited 2019 